\title{
Physical-Statistical Modeling of Dynamic Indoor Power Delay Profiles
}

\author{
Evgenii Vinogradov, Aliou Bamba, Wout Joseph, Claude Oestges
}

\begin{abstract}
This paper presents a physical-statistical radio channel power delay profiles model for room-toroom communication systems combining the Room Electromagnetic Theory for modeling deterministic channel components with a geometry based stochastic channel model with time-variant statistics for modeling stochastic components. The deterministic channel component, i.e., mean power delay spectrum, is comprised of specularly reflected paths plus diffuse components due to scattering and diffraction. The specular components are modeled with a set dirac function whereas the diffuse components modeling approach is a room electromagnetic theory-based model. Dynamic indoor communication channels are characterized by a non-stationary time- and delay fading process due to changes in the environment. We analyze and model the time-delay variability of channels using K-factor for small-scale variations and the t-location scale distribution parameters for large-scale variations. It turns out that these parameters cannot be assumed to be constant in time and delay. After modeling of time-delay variations of the first order statistics, we generate channel realizations with appropriate second order statistics. As the result, the presented model enables to describe the evolution of the power delay profile in the time domain.
\end{abstract}

\section{Index Terms}

E. Vinogradov is with KU Leuven, Department of Electrical Engineering - ESAT, Leuven Belgium.

C. Oestges is with the Electrical Engineering Department, Institute for Information and Communication Technologies, Electronics and Applied Mathematics (ICTEAM), Université catholique de Louvain, 1348 Louvain-la-Neuve, Belgium (e-mail: Evgenii.Vinogradov@UCLouvain.be)

A. Bamba is affiliated with Ecole Supérieure Africaine des TIC (ESATIC), Treichville, Km4 Bd de Marseille, Abidjan, Cote d'Ivoire and Ghent University.

W. Joseph is with Ghent University/IMEC, Technologiepark 159052 Gent, Belgium

This research is funded by the Interuniversity Attraction Poles Programme 7/23 BESTCOM initiated by the Belgian Science Policy Office. It is also carried out in the framework of the FP7 Network of Excellence NEWCOM\#, COST Action CA15104 IRACON. 
Indoor propagation, Fading channels, Multipath channels, Propagation measurements, Time-varying channels, Room Electromagnetic theory.

\section{INTRODUCTION}

Predicting the parameters of indoor wireless channels is important for the testing and the eventual rollout of mobile-to-mobile communication systems. The a-priori knowledge of the power - or energy - density of the propagating electromagnetic fields (EMF) throughout a given environment is required for coverage prediction. Besides, the dispersiveness of the radio channel determines the maximum achievable transmission free of inter-symbol interference (ISI). The received power and dispersiveness of the multipath in a wireless channel can be typically predicted from the power delay profile (PDP).

Deterministic approaches such as ray tracing methods [1], [2], [3], [4], [5], [6] have been used to predict the characteristics of radio channels. Although excellent agreement can be obtained with such numerical approaches, high computational cost might make them less attractive. Furthermore, the cost increases as well when the room dimensions and/or frequency increase.

Indoor wireless channel modeling has been empirically addressed in [7], [8], [9]. These studies investigated indoor path loss and/or fading models around 1.8 and $5.2 \mathrm{GHz}$. Empirical path loss models for in-room, room-to-corridor, and room-to-room scenarios have also been investigated [10]. The one-slope log-distance, dual-slope log-distance and attenuation factor model are used to estimate the path loss in in-room, room-to-corridor, and room-to-room scenarios, respectively.

Most previous works e.g., [11], [12] have modeled indoor channels for static scenarios or so-called nomadic scenarios (fixed nodes, moving obstacles). In [11] the large-scale fading, Kfactor, and delay spread are found to be correlated lognormal variables, whereas in [12] fading is found to be SOSF (Second Order Scattering Fading [13]) distributed.

Dynamic models of indoor wireless channels were proposed in [14], [15], [16], where the motion of communication nodes and obstacles (such as people) is taken into account. Whereas models in [12], [15], [16] are narrowband, [14] predicts the time-varying PDP as a weighted sum of cluster functions.

It was shown in [17] that non-stationarity is an important issue for indoor channels. Hence, the effects brought by nodes' mobility must be considered in the channel modeling. Nonstationary channel statistics for wireless vehicle-to-vehicle (V2V) and vehicle-to-infrastructure 
(V2I) communications were investigated in [18], where a bi-modal Gaussian mixture distribution was used to model the statistics of time-frequency variant K-factor.

Indoor channels do not experience interactions with highly mobile objects, however even a slow motion can cause significant change of the environment and, consequently, changes of the channel statistics. Since deterministic approaches are computationally expensive, a statistic approach to model room-to-room wideband channels with time- and delay-variant statistics under mobile conditions is missing.

In this paper, we propose a physical-statistical technique combining the Room Electromagnetic (REM) Theory for modeling deterministic channel components with a geometry based stochastic channel model with time-variant statistics for modeling stochastic channel components. The method enables the reconstruction of the PDP in a room-to-room dynamic scenario, including effects introduced by the mobility of the communication nodes. The main contributions are as follows:

- The prediction of the specular reflections is improved as the motion of the transceivers is accounted for.

- We analyze and model the time-delay variations of indoor channels statistics based on measurements. The combination of extracted first- and second-order statistics enables to implement a channel impulse response (CIR) generator.

- To model the sudden changes of fading statistics, we use a three-step approach: (i) for reflecting the sudden changes of the statistics, we use a hidden Markov model [19] parameterized from measurements, (ii) the impacts of the line-of-sight (LOS) term and the Rayleigh component are modeled according to a Extreme Value distribution, fitting the measurements, (iii) fading realizations are generated [20].

- A model for large scale variations of the CIR is proposed.

The paper is organized as follows: Section II presents the measurement setup, including the scenario, sounding equipment and antennas. It also outlines the data post- processing approach and the channel representation. Section III presents the modeling of deterministic channel components, while Section IV describes analyzed experimental results as well as the modeling of time-variant components. The global model is validated in Section V. Finally, conclusions are drawn in Section VI. 
TABLE I

DISTANCE BETWEEN THE NODES

\begin{tabular}{c|ccccc}
\hline Node & $\mathrm{Rx} 1$ & $\mathrm{Rx} 2$ & $\mathrm{Rx} 3$ & $\mathrm{Rx} 4$ & $\mathrm{Rx} 5$ \\
\hline $\mathrm{Tx} 1$ & $4.9 \mathrm{~m}$ & $6.5 \mathrm{~m}$ & $4.6 \mathrm{~m}$ & $5.6 \mathrm{~m}$ & $7.4 \mathrm{~m}$ \\
\hline \hline Node & $\mathrm{Rx} 6$ & $\mathrm{Rx} 7$ & $\mathrm{Rx} 8$ & $\mathrm{Rx} 9$ & $\mathrm{Rx} 10$ \\
\hline Tx1 & $9.7 \mathrm{~m}$ & $12 \mathrm{~m}$ & $12.1 \mathrm{~m}$ & $9.2 \mathrm{~m}$ & $7.6 \mathrm{~m}$ \\
\hline
\end{tabular}

\section{Measurements And Methodology}

\section{A. Environment}

This paper is based on channel measurements carried out at the Universite catholique de Louvain (UCL), Louvain-la-Neuve, Belgium, in fall 2014. The investigated environment was located on the second floor of an office building, and consisted of two adjacent typical office rooms separated by a brick wall, as illustrated in Fig. 1. Circles indicate mobile receivers Rx1 to Rx10 (moving in random directions over a small area within a square of $1 \mathrm{~m}^{2}$ at walking speed, $v \approx 1 \mathrm{~m} / \mathrm{s}$ ), whereas the square indicates the static transmitter (Tx). The separations between all nodes are listed in the Table I.

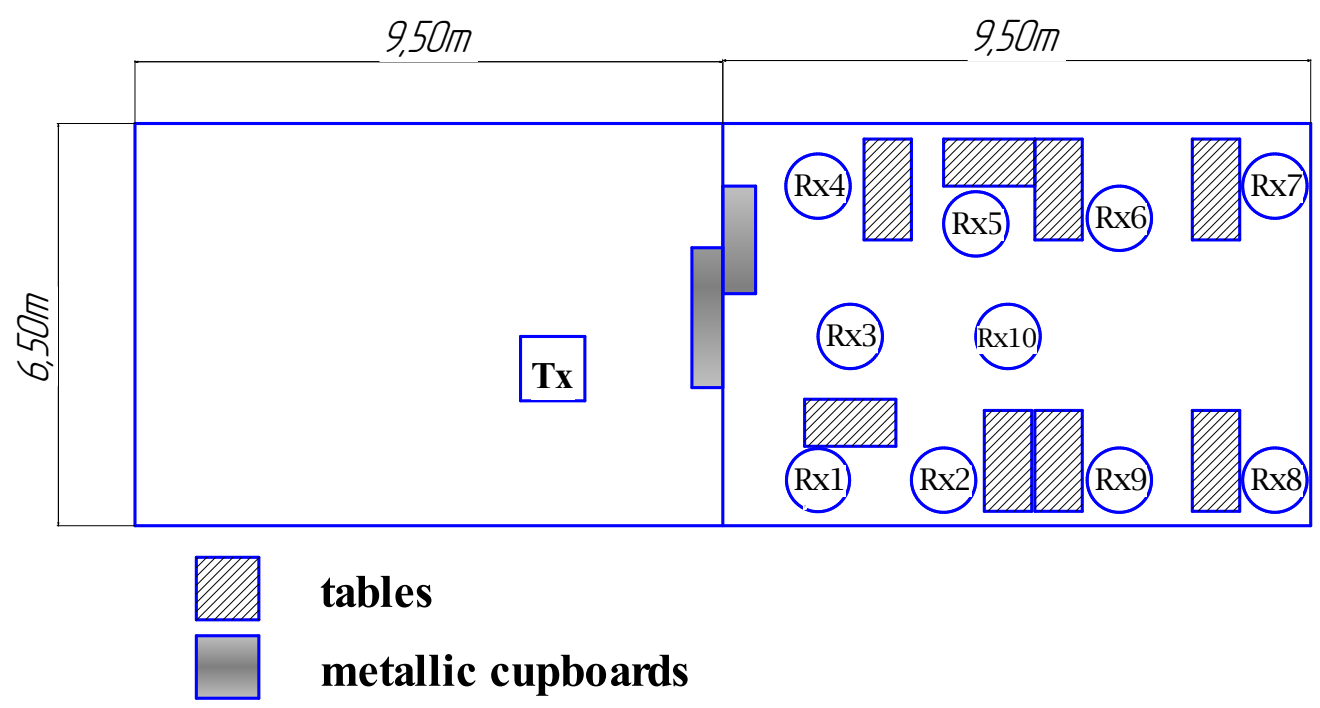

Fig. 1. Floor-plan of measurements 
TABLE II

MEASUREMENT PARAMETERS

\begin{tabular}{cc}
\hline Parameter & Value \\
\hline Center frequency, $f_{c}$ & $3.8 \mathrm{GHz}$ \\
Transmit power, $P_{t x}$ & $23 \mathrm{dBm}$ \\
Measurement bandwidth, $B$ & $200 \mathrm{MHz}$ \\
Recorded delay chips, $C$ & 2048 \\
Recorded time samples, $N_{s}$ & 6000 \\
Measurement duration, $T$ & $60 \mathrm{~s}$ \\
Code length, $l$ & $20.47 \mu \mathrm{s}$ \\
\hline
\end{tabular}

\section{B. Equipment}

The measurements were carried out with UCL/ULB Elektrobit PROPSound ${ }^{\mathrm{TM}}$ Channel Sounder (CS) at a carrier frequency of $f_{c}=3.8 \mathrm{GHz}$. The nodes were connected with the channel sounder using long low-loss RF cables of equal length. These cables had excellent RF stability, even when they were slightly bent or moved during the measurements. At the nodes, we used custom-made dipole antennas with a gain of $\mathcal{G}=1.75 \mathrm{~dB}$, an omnidirectional radiation pattern in the horizontal plane and $3 \mathrm{~dB}$ beamwidth of $80^{\circ}$ in the vertical plane (see [16]). The channel sounder used long pseudo-noise (PN) sequences to estimate the impulse response of the radio channels between Tx and Rx nodes. The measurement parameters are summarized in Table II. The recorded channel impulse response is denoted by $h_{j}(t, \tau)$, where $t$ denotes the time, $\tau$ denotes the delay, and $j$ denotes the link index (a link joining one transmit node to one receive node).

\section{Channel representation}

The methodology proposed here is based on $i$ ) the prediction of the deterministic PDP (in static scenario), and $i$ ) the prediction of the (stochastic) large- and small-scale fading statistics. The small-scale fading statistics [15], [16] describe the variations - around the average power level - caused by random motions of the transceivers. The general methodology to reconstruct the PDP is illustrated in Fig. 2.

Indoor channel measurements have shown that the PDP averaged over time consists of two parts: a primary part and a reverberant part [21], [22], [23]. The complete PDP is the superposition of 
the primary and reverberant components, the large-scale fading which affects only the primary component, and the small-scale fading.

$$
\operatorname{PDP}(t, \tau)=\left(S(t) \cdot \operatorname{PDP}_{p r i}(\tau)+\operatorname{PDP}_{r e v}(\tau)\right) \cdot|G(t, \tau)|^{2}
$$

where $\mathrm{PDP}_{\text {pri }}, \mathrm{PDP}_{r e v}, S$ and $G$ are the (deterministic) primary component, (deterministic) reverberant component, the temporal large-scale fading (in power) and small-scale fading (in amplitude), respectively. All components in (1) are expressed in a natural scale, i.e not logarithmic.

\section{DETERMINISTIC PDP COMPONENTS}

Step A of Fig. 2 is explained in this section. Assume a transceiver located at a given position in an indoor environment. If a signal pulse is transmitted in the room, the receiver records first the line-of-sight (LOS) component if present. Afterwards, few specular paths due to reflections off the walls, ceiling, or floor arrive at the receiver. For a given position of the transceiver, the specular paths - LOS included - arrive at the receiver side with incident angles characterized by an elevation angle $\theta$ and an azimuth $\varphi$, which are dependent on the transceiver's position, the room layout's, the furniture, and so on. Therefore, the arrival angles of specular multipath are deterministic, and do not contribute to the establishment of the diffuse fields. During this transient or primary state, the energy of the EMF is not uniform in the room as the contributions of the specular paths differ from one direction to another one [24]. Besides the specular reflections, there are also propagation phenomena such as scattering, and/or diffraction that establish the diffuse fields. The transient and the reverberant state are shown in Fig. 3 and the corresponding powers

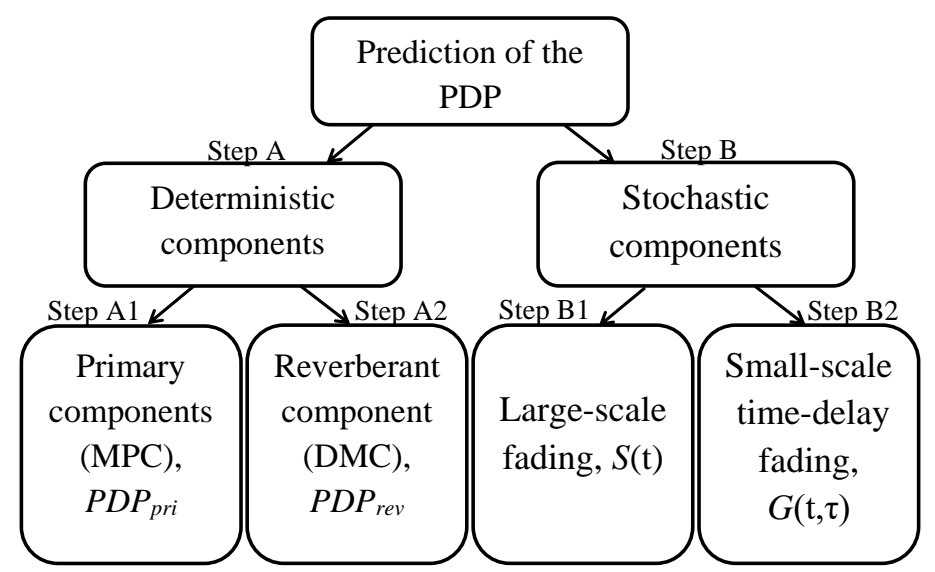

Fig. 2. Schematic of the methodology. The different steps are further explained. 


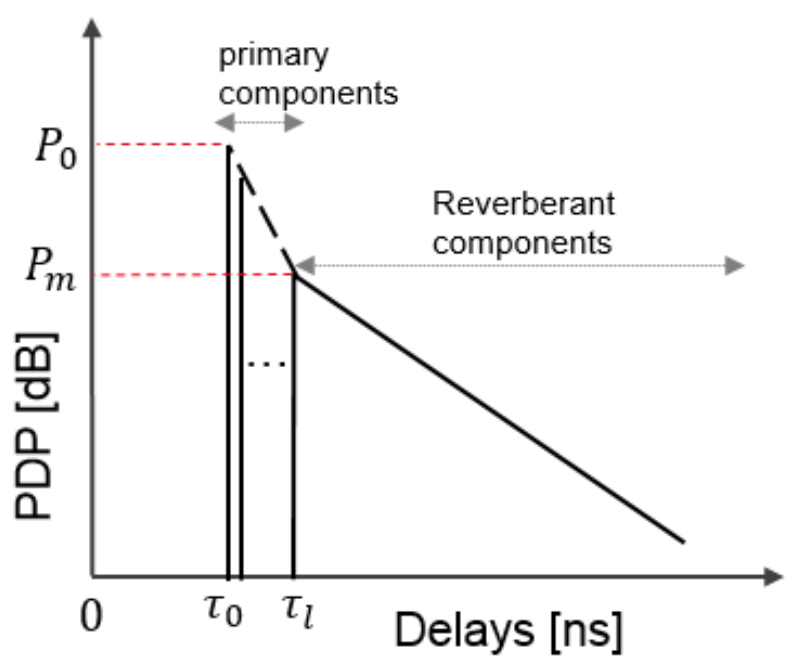

Fig. 3. Modeling of the PDP in a reverberant environment. $\tau_{0}$ is the arrival delay of the first or strongest component and $\tau_{l}$ is the end (resp. beginning) of the primary (resp. reverberant) component

are denoted as primary and reverberant components, respectively. The power delay spectrum (or the deterministic components) is the superposition of both the primary and reverberant component and is expressed as follows:

$$
\mathrm{PDP}=\mathrm{PDP}_{p r i}+\mathrm{PDP}_{r e v}
$$

In dB-scale, the reverberant part is characterized by the slope in the tail of the PDP (Fig. 3), and is independent on the transceivers location. However, the primary part does depend on the location of the transceiver. For instance, for a short (resp. long) Tx-Rx separation, the transient state will last longer (resp. shorter) than if the Tx-Rx distance was longer (resp. shorter). Moreover, the transceiver's location will influence the power level of the primary components, which consists of the LOS if present and a number of specular reflections.

\section{A. Modeling of the Primary Components (Step A1)}

The existence of primary components in indoor environments is well acknowledged in the literature [21], [22], [23], [25]. However, the primary components is usually modeled as a delta function, i.e., one specular component:

$$
\operatorname{PDP}_{p r i}(\tau)=P_{0}\left(\tau_{0}\right)^{n} \delta\left(\tau-\tau_{0}\right)
$$


where $P_{0}\left(\tau_{0}\right)$ is the power of the primary or strongest component arriving with a delay $\tau_{0}$, and $n$ is the path loss exponent. Parameter $n$ is determined by fitting the experimental path gain obtained at different $\mathrm{Tx}-\mathrm{Rx}$ distances.

The modeling of the primary components with one delta function fits better for longer Tx-Rx separation, than for shorter ones. This is confirmed by several experimental measurements in indoor environments, e.g., [26], [27], [25] and simulations [28] where the contribution of the primary component in the total power becomes negligible for larger Tx-Rx separation. The modeling of the primary components is improved with a set of delta functions:

$$
\operatorname{PDP}_{p r i}(\tau)=\sum_{m=0}^{M} P_{0}\left(c_{0} \tau_{0}+m c_{0} \tau_{c}\right)^{n} \delta\left(\tau-\tau_{0}-m \tau_{c}\right),
$$

where $M$ is the reflection order and $\tau_{c}$ is the characteristic time-delay that is required before a given ray makes one reflection in the room [29]. It has been shown that a ray undergoing $m$ reflection(s) off the surfaces before arriving at the receiver has an arrival delay of $\tau=m \tau_{c}$, where $\tau_{c}$ depends only on the dimensions of the room and is expressed as [29]:

$$
\tau_{c}=\frac{8 \mathrm{~V}}{c_{0} S}
$$

where $V, S, c_{0}$ are the room volume, the total surface area of the room, and the speed of light in free space.

In the model we consider reflections up to the second order, i.e., the primary component consists of the LOS component, the first and second order reflected paths. The average power of a ray bouncing $m$ time(s) ( $m \in\{0,1,2\})$ is approximated by the free-space gain equation:

$$
P_{0}\left(d_{0}+m c_{0} \tau_{c}\right)^{n}=N \frac{\alpha^{m}}{\left(d_{0}+m c_{0} \tau_{c}\right)^{n}}
$$

where $N$ is a constant depending on antenna properties, frequency, and transmitted power, and $\alpha$ is the average reflection coefficient of the wall surfaces.

\section{B. Modeling of the Reverberant Component (Step A2)}

The modeling of the reverberant component is straightforward and is based on the Room Electromagnetic theory [23]. The reverberation time of the considered room, and the power corresponding to the beginning of the reverberant components $\left(P_{m}\right.$ in Fig. 3$)$ are the required parameters to model the reverberant component. An empirical formula to determine the reverberation time as a function of the frequency is derived in [30] and can be used to assess the 
reverberation time without resorting to measurements. However, in this study, we consider a room-to-room scenario as illustrated in Fig. 1. The expressions of the reverberation time for in-room and room-to-room communications are derived in [27].

The power level $P_{m}$ shown in Fig. 3 is the same as the primary component at $\tau=\tau_{l}$ :

$$
P_{m}=\operatorname{PDP}_{p r i}\left(\tau_{l}\right)
$$

If we include the time-delay dependency in the model, the reverberant component is given by [21], [22], [23]:

$$
\begin{aligned}
\operatorname{PDP}_{r e v}(\tau) & =P_{m} e^{-\frac{\left(\tau-\tau_{l}\right)}{\tau_{r}}} & & \text { if } \tau \geq \tau_{l} \\
& =0 & & \text { otherwise }
\end{aligned}
$$

where $\tau_{r}$ is the reverberation time of the environment. The reverberation time is the decay rate of the diffuse energy and has been experimentally and numerically investigated in various studies [22], [28], [27], [23]. The reverberation time for an in-room scenario can be determined via the following formula [30]:

$$
\begin{aligned}
\tau_{r}(f) & =\frac{V}{2 \pi f A}\left(0.473 f^{3}-24.9 f^{2}+321 f-254\right) \\
& =\frac{4 V}{c_{0} A \eta(f)}
\end{aligned}
$$

where $\tau_{r}$ and $2<f<10$ are the reverberation time (ns) and the frequency (GHz), respectively; $\eta(f)$ is the average fraction of the energy absorbed by the surfaces in the room at the frequency $f$ and $A$ is the wall's surface. Once the reverberation time for in-room scenario is determined, its value for the adjacent room scenario (room-to-room) is determined by [27]:

$$
\tau_{r, a}=\frac{\tau_{r}}{1-\frac{c_{0} \tau_{r} A_{12}}{4 V}},
$$

where $\tau_{r, a}, \tau_{r}$, and $A_{12}$ are the reverberation time in adjacent room (i.e., the $\mathrm{Tx}$ and the $\mathrm{Rx}$ are in adjacent rooms), the reverberation time when the transceivers are in the same room (in-room scenario), and the surface of the wall separating the adjacent rooms, respectively. The average fraction of absorbed energy (hence the average reflection coefficient, i.e., $\alpha=1-\eta$ ) is further determined from (10). 


\section{Time-Delay VARiant Channel Components}

The modeling approach for the stochastic channel components (step B of Fig. 2) is explained in this section. As mentioned above, the stochastic channel components can be expressed as the product (when expressed in natural scale) of the temporal large-scale variations $S(t)$ and time-delay variant small-scale variations $G(t, \tau)$ :

- Temporal large-scale variations $S(t)$ are introduced by mobility of scatterers and slow changes of the environment. Only primary components (see Fig. 3) are affected.

- Time-delay small-scale variations $G(t, \tau)$ (in natural scale) caused by multipath interference resulting from the small-scale motions of the stations and/or the environment. Both primary and reverberant components are influenced by these variations

$$
G_{j}(t, \tau)=\frac{h_{j}(t, \tau)}{\sqrt{\mathrm{PDP}_{a v, j}(\tau) \cdot S_{j}(t)}},
$$

where $\operatorname{PDP}_{a v, j}(\tau)$ is a PDP for channel $j$ averaged over the whole measurement duration, the

small-scale fading amplitude is then simply defined as $g=|G|$. Analogous to the terminology used for narrowband channels, for channel $j$, let us denote $S_{j}(t)$ and $G_{j}(t, \tau)$ as large- and small-scale fading, respectively.

Figs. $4 a, b, c$ show measured changes of the received power, measured PDP that is constant over time for a given link and time evolution of large- and small-scale variations for a fixed delay, respectively.

\section{A. Large-Scale Fading Characterization and Modeling (Step B1)}

To extract non-deterministic components of the channel, first, the measured $\mathrm{PDP}_{a v}$ has to be subtracted from the considered channel $P_{j}(t, \tau)=\left.10 \log \left|h_{j}(t, \tau)\right|^{2}\right|_{d B}-\left.\mathrm{PDP}_{a v, j}(\tau)\right|_{d B}$. Next, to estimate large-scale fading, we average out the small-scale fading by using a moving window spanning $T_{a v}=1.5 \mathrm{~s}$ ( see Fig $4 \mathrm{c}$ ), so that the time-variant average power can be written at discrete time $t$ as

$$
S_{j}[t]=\frac{1}{T_{a v} \cdot \tau_{l}} \sum_{t^{\prime}=t-T_{a v} / 2}^{t+T_{a v} / 2} \sum_{\tau^{\prime}=0}^{\tau_{l}} P_{j}\left(t^{\prime}, \tau^{\prime}\right) .
$$

The choice of $T_{a v}$ is such that the small-scale fading is averaged out, while still following the slow variations induced by the motion of the nodes and/or people. Different sizes of the window have been checked and the window span has been chosen to be equivalent to 20 wavelengths. This size of the sliding window was also used in [31], [32]. 


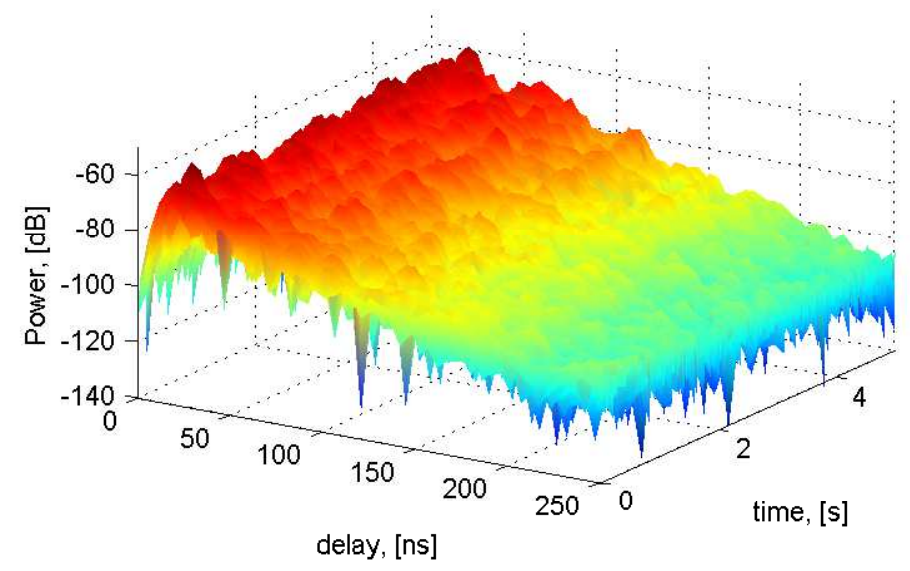

(a) Time evolution of relative power

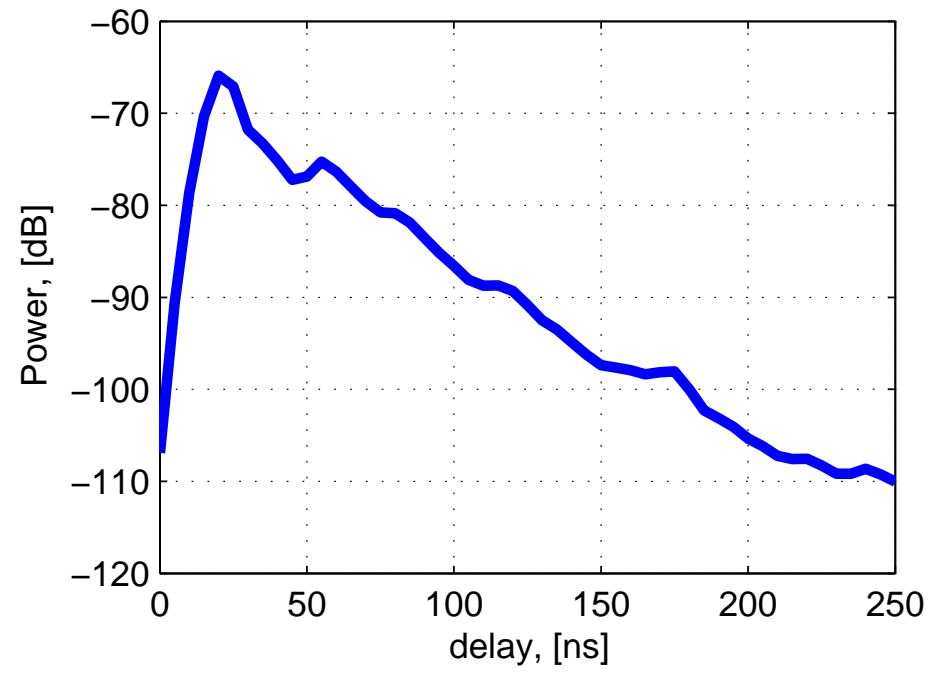

(b) Power delay profile

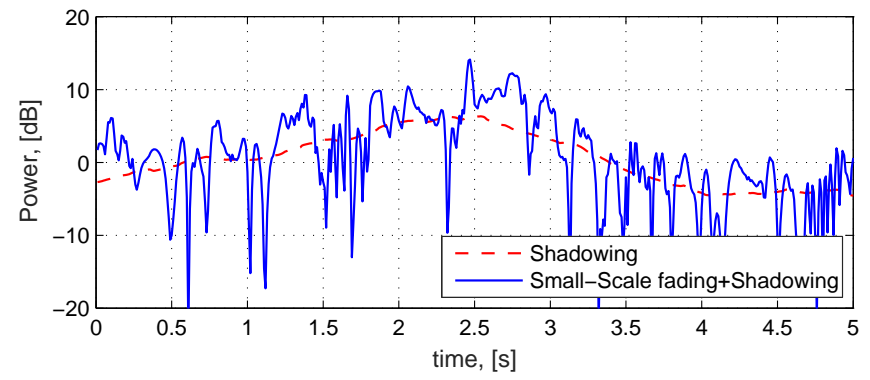

(c) Time-variant components of the channel (Primary component, fixed delay)

Fig. 4. Channel components, Tx-Rx2

When the static $\mathrm{PDP}_{a v, j}$ is removed and time-delay small-scale fading is averaged out, the remaining variations are due to large-scale fading, as detailed earlier. Note, that further modeling 
and analysis for the large-scale fading $S$ is done in $\mathrm{dB}$. It turns out that the large-scale fading in the investigated measurement setup is described by a t-location scale distribution

$$
p\left(x \mid \nu, \mu, \sigma_{\tilde{s}}\right)=\frac{\Gamma\left(\frac{\nu+1}{2}\right)}{\Gamma\left(\frac{\nu}{2}\right) \sigma_{\tilde{s}} \sqrt{\pi \nu}}\left(1+\frac{1}{\nu}\left(\frac{x-\mu}{\sigma_{\tilde{s}}}\right)^{2}\right)^{-\frac{\nu+1}{2}}
$$

with zero-mean $(\mu=0)$ by definition, $\nu=8$ degrees of freedom and a scale parameter $\sigma_{\tilde{s}}=2.4$. This distribution has been used previously to describe large-scale fading in [16] and results from compounding a normal distribution with mean $\mu$ and unknown variance, with an inverse gamma distribution placed over the variance with parameters $\alpha_{\Gamma}=\nu / 2$ and $\beta_{\Gamma}=\nu \sigma_{\tilde{s}}^{2} / 2$. In other words, the dynamic large-scale fading is assumed to have a Gaussian distribution with an unknown variance distributed as inverse gamma, and then the variance is integrated out as shown in [33].

The dynamic large-scale fading autocorrelation function (ACF) is well approximated by a decreasing exponential process, whose decay time $\tau_{\tilde{s}}=8.4 \mathrm{~s}$. Hence, an autoregressive process can be used to generate autocorrelated dynamic large-scale fading values:

$$
\left.S[t]\right|_{d B}=e^{-\Delta t / \tau_{S}} S[t-1]+\sqrt{1-e^{-2 \Delta t / \tau_{S}}} x_{S}[t]
$$

where $x_{S}$ is a t-location scale distributed variable generated independently for each discrete time instant $t$ [33] and $\Delta t$ is the sampling rate which equals $1.5 \mathrm{~s}$.

No correlation between dynamic large-scale fading and the small-scale fading states has been found, so that we can combine independently simulated dynamic large-scale fading and smallscale fading realizations.

\section{B. Small-Scale Fading Modeling (Step B2)}

First, the Generalized Local Scattering Function (GLSF) [34] was obtained using the estimator presented in [35]. The window lengths in time and frequency were chosen to be $N_{w, t}=N_{w, f}=$ 32. In the GLSF estimation, the windows were created by a discrete prolate spheroidal sequence, the number of windows was set to $I=J=3$ as proposed in [36]. Next, the collinearity was calculated and the threshold $c_{t h}=0.9$ was used to estimate stationarity regions as shown in [37]. It turns out that the maximum stationarity bandwidth is $100 \mathrm{MHz}$. Since the experimental delay resolution is $5 \mathrm{~ns}$, we can say that the amplitudes of two realizations in neighboring delays are correlated. 
TABLE III

PROBABILITIES OF THE SUBSETS AND TRANSITION PROBABILITIES

\begin{tabular}{|c|c|c|c|}
\hline \multirow{2}{*}{ Subset } & \multirow{2}{*}{ Probability } & \multicolumn{2}{|c|}{ Transitions } \\
\hline & & Rician & Rayleigh \\
\hline Rician & 0.65 & 0.84 & 0.16 \\
\hline Rayleigh & 0.35 & 0.85 & 0.15 \\
\hline
\end{tabular}

To extract first-order statistics of time-delay fading, the whole delay range is first partitioned into bins of $\tau_{b}=10 \mathrm{~ns}$ ( 2 delay chips) each. It turns out that complex channel impulse responses fade out to the noise level for delays larger than $250 \mathrm{~ns}$ (50 delay chips), so that we can use only first 25 delay bins. This leads to a total number of $B=25$ bins per time instant and link. Next, $40 \times 25$ blocks of $150 \times 2$ realizations (corresponding to $\frac{T}{T_{a v}}$, where $T_{a v}=1.5 \mathrm{~s}$ or 20 wavelength and $T=60 \mathrm{~s}$ ) can be used to extract statistics (using Least Squares Fitting).

Note that we can normalize fading so that $\mathbb{E}\left\{g^{2}\right\}=1$, the expectation being taken over time and delay.

1) First-Order Fading Statistics: As shown in [16], we can expect small-scale fading within a bin to be Rician or Rayleigh distributed for single mobile scenarios. The time-delay varying $K$-factor can be estimated from experimental time-delay fading realizations $g_{j}(t, \tau)$ by fitting the Rice probability density function. $40 \times 25$ realization blocks have been used. Extracted probabilities of the subsets (Rician and Rayleigh) and transition probabilities are shown in Table III.

Temporal transitions between different fading states can be modeled by means of a hidden Markov model (HMM) [19]. Usually, the system being modeled by a HMM is assumed to be a Markov process with unobserved (hidden) states. Since only two distributions of small-scale fading are observed, we model the transitions between the subsets using a simple two-state HMM: (i) Rican fading and (ii) Rayleigh fading. Consequently, the HMM is parameterized from the measurements.

To simplify the usage of first- and second- order statistics derived in [16], let us denote:

$$
\begin{gathered}
\omega_{0}=\sqrt{\frac{K}{1+K}}, \\
\omega_{1}=\sqrt{1-\frac{K}{1+K}},
\end{gathered}
$$




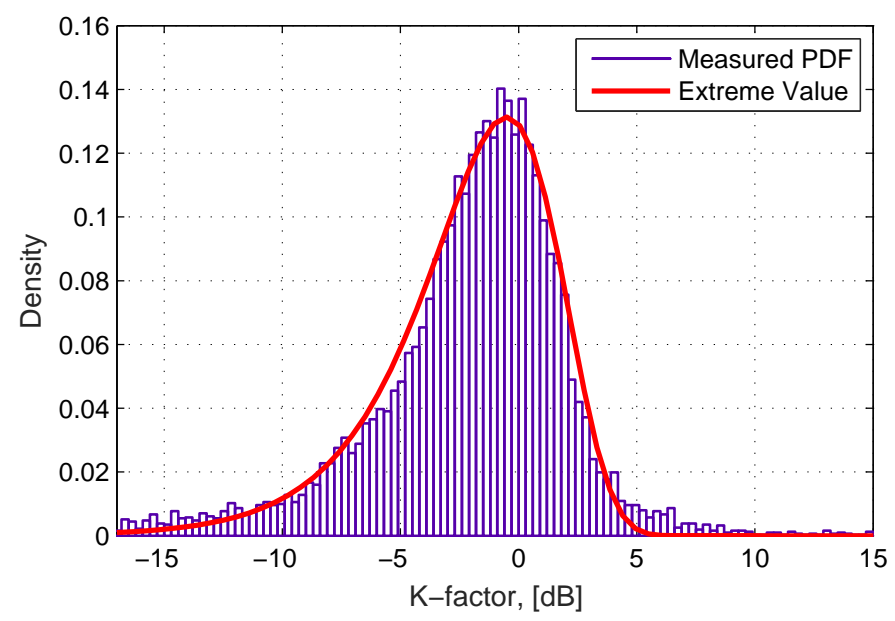

Fig. 5. Distribution of the Rician $K$-factor

where $K$ is the estimated $K$-factor (in $\mathrm{dB}$ ); $\omega_{0}$ and $\omega_{1}$ describe the impact of the LOS term and Rayleigh component respectively.

The distribution of the $K$-factor for the Rician subset can be modeled by Extreme Value distribution,

$$
p_{e v}(x \mid \mu, \sigma)=\sigma^{-1} e^{\left(\frac{x-\mu}{\sigma}\right)} e^{\left(-e^{\left(\frac{x-\mu}{\sigma}\right)}\right)}
$$

when the $K$-factor expressed in decibels (see Fig 5). The temporal autocorrelations of the measured $K$-factor is a decreasing exponential function . Hence, the model presented in [16] (i.e. an autoregressive process) can be used to generate autocorrelated values of the $K$-factor over time samples $(t=[1, \cdots, T])$ :

$$
\left.K[t]\right|_{d B}=\left.e^{-\Delta t / \tau_{K}} K[t-1]\right|_{d B}+\sqrt{1-e^{-2 \Delta t / \tau_{K}}} x_{K}[t],
$$

where $x_{K}$ is a time series of length $T$, whose values are drawn independently from the appropriate distribution. The parameter $\tau_{K}=2.5[s]$ is extracted from measurements.

It turns out that the first order statistics are not correlated over delay bins, consequently, time-variant fading within a bin can be modeled independently from neighboring bins.

2) Second-Order Fading Statistics: The room to room environment can be approximated by a double-ring representation [38] as shown in [16]. The correlation functions of the fading can 
TABLE IV

MEASURED PARAMETERS

\begin{tabular}{cc}
\hline Parameter & Value \\
\hline$\omega_{D B}$ & $\frac{2 \omega_{1}}{3}$ \\
$\omega_{S B T}=\omega_{S B R}$ & $\frac{\omega_{1}}{6}$ \\
$\mathrm{R}$ & $3 \mathrm{~m}$ \\
$f_{m}$ & $13.8 \mathrm{MHz}$ \\
\hline
\end{tabular}

be expressed as [16]

$$
\begin{aligned}
& R_{G_{i} G_{i}}(\tau)=R_{G_{q} G_{q}}(\tau)=R_{G G}(\tau)= \\
& =\mathbb{E}\left[G_{q}(t) G_{q}(t+\tau)\right] \\
& =\omega_{0}^{2} \cos \left(2 \pi \tau\left(f_{m} \cos \Theta\right)\right) \\
& +\left(\omega_{D B}^{2}+\omega_{S B R}^{2}\right) \cdot J_{0}\left(2 \pi f_{m} \tau\right) \\
& +\omega_{S B T}^{2} \cdot J_{0}\left(\frac{R^{2}}{D^{2}} \pi f_{m} \tau\right) \\
& R_{G_{i} G_{q}}(\tau)=-R_{G_{q} G_{i}}(\tau)=0
\end{aligned}
$$

where $f_{m}, \Theta, R$ and $D$ denote the Doppler frequency caused by motion of the mobile node, the angle of the motion, radius of the ring which is formed by scatterers located around the static node and the distance between Tx and Rx, respectively.

Small-scale fading realizations used to extract the parameters $\omega_{0}$ and $\omega_{1}$ were analyzed to estimate the parameters of second order statistics. First, the Doppler Spectrum was calculated. Next, the Doppler frequency was estimated so that we could calculate the radii using measured Doppler spectrum and the theoretical one. Finally, estimated parameters $\left(R, f_{m}\right.$ and the components weights) were averaged over all channels and samples and bins. The summary of the analysis is listed in Table IV.

\section{Time-Series Modeling}

Summarizing, we propose to model channels with time-variant statistics and a static PDP as shown in Fig. 6. We start with a random initial state of the HMM. Next, using the corresponding 


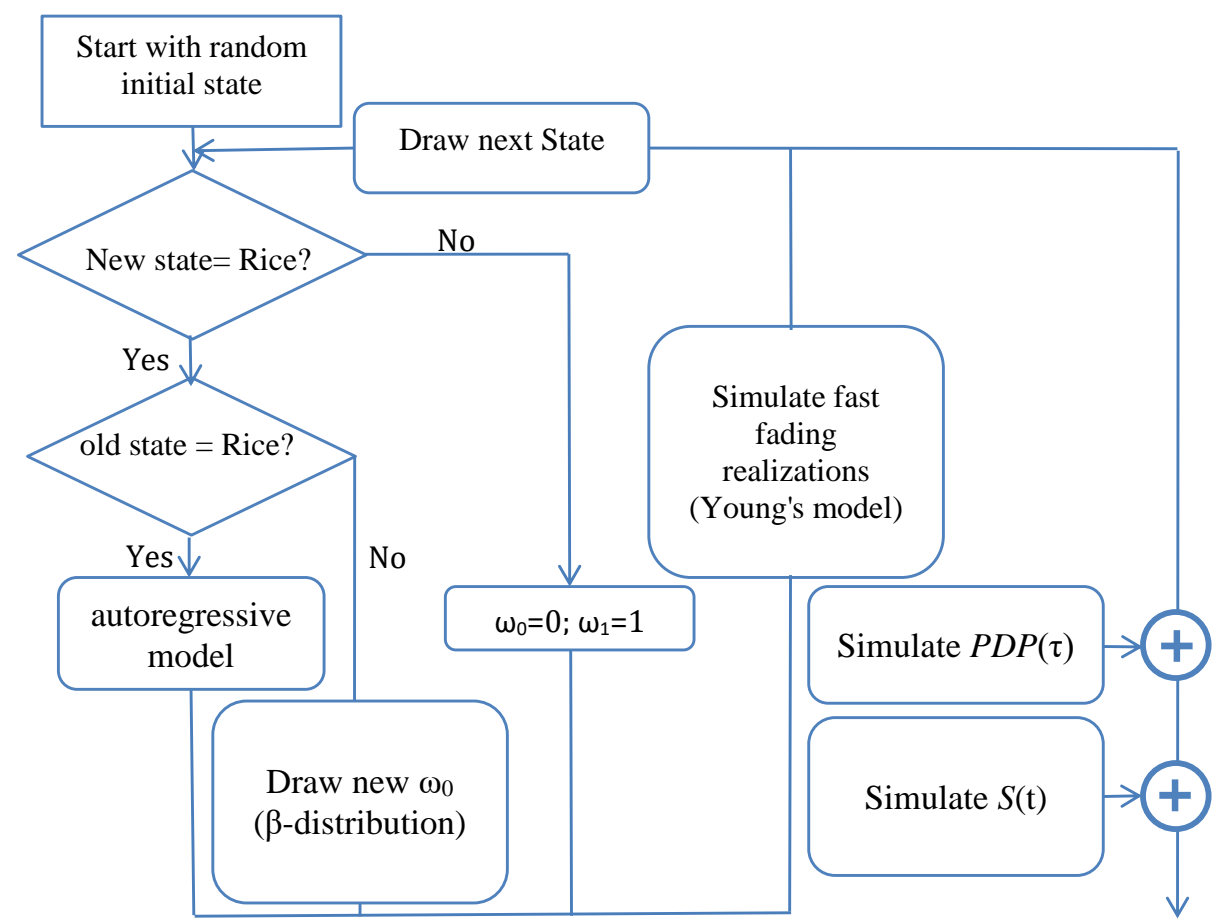

Fig. 6. Flow diagram of the complete model (Steps A+B)

distribution, we draw the weights $\left(\omega_{0}, \omega_{1}\right)$. Using a weighted combination of correlated smallscale fading realizations generated by the model $^{1}$ described in [20], we obtain a pre-defined number of correlated complex fading realizations $G(t)$. Next, the $P D P(\tau)$ corresponding to the considered delay and the dynamic large-scale fading are added to the small-scale fading realizations. Next, the state of the HMM is updated. If the state is unchanged, we use the autoregressive model described by (19) to generate a new value of $\omega_{0}$ for the Rician subset or we keep the constant values of the parameters for the Rayleigh subset. If the state is modified, depending on the subset, we generate the weights $\omega_{0}$ and $\omega_{1}$ using the corresponding distribution or we set fixed values (for Rayleigh). For the next delay bin the algorithm should be repeated. Note that the duration of one state equals $1.5 \mathrm{~s}$.

${ }^{1}$ Filter coefficients used in the model are specified by appropriate first- and second-order statistics derived in [16]. 


\section{Simulation Results and Validation}

\section{A. Reconstructed Power Delay Profiles}

The deterministic modeled PDP do not vary with time. For a given position, the variation in the measured PDP is due to the small-scale fading. The deterministic models are presented in Figs. $7 a, b$. Measured time-variant PDPs are shown in Figs. $7 c, d$. The complete model is the aggregate of the large- and small-scale fading levels and the deterministic model for the considered nodes $(\mathrm{Rx} 1,7)$. Example of the total PDP models in the dynamic scenario are shown in Figs. $7 e, f$.

Given the room dimensions in Fig. 1, and by applying (9), we obtain a reverberation time of about $\tau_{r, a}=20.8 \mathrm{~ns}$ for the in-room scenario. In this study, Tx and $\mathrm{Rx}$ are located in adjacent rooms (see Fig. 1). Therefore, we apply (11) and obtain a theoretical reverberation time of about $\tau_{r, a}=24.8 \mathrm{~ns}$ for the adjacent room scenario.

TABLE V

EXPERIMENTAL VALUES OF THE REVERBERATION TIME FOR THE DIFFERENT NODES IN THE ADJACENT ROOM SCENARIO

\begin{tabular}{c|ccccc}
\hline Node & $\mathrm{Rx} 1$ & $\mathrm{Rx} 2$ & $\mathrm{Rx} 3$ & $\mathrm{Rx} 4$ & $\mathrm{Rx} 5$ \\
\hline$\tau_{r, a}(\mathrm{~ns})$ & 21.85 & 21.45 & 25.10 & 28.20 & 22.80 \\
\hline \hline Node & $\mathrm{Rx6}$ & $\mathrm{Rx} 7$ & $\mathrm{Rx} 8$ & $\mathrm{Rx} 9$ & $\mathrm{Rx} 10$ \\
\hline$\tau_{r, a}(\mathrm{~ns})$ & 19.90 & 20.55 & 20.75 & 20.55 & 22.10 \\
\hline
\end{tabular}

Experimental values of the reverberation time at different nodes are shown in Table V. On average, an experimental reverberation time of $\tau_{r, a}=22.3 \mathrm{~ns}$ and a standard deviation $\sigma=2.5 \mathrm{~ns}$ are obtained. The relative difference between the experimental and theoretical reverberation time is about $11.20 \%$, indicating good agreement between the predicted and measured reverberation time [30]. The formula overestimates the reverberation time value because of the presence of furniture in the room. Only walls, floor and ceiling are indeed considered; hence, this will lower the experimental reverberation time value compared to the predicted one.

\section{B. Validation}

The validation is an important step in any model development. To validate the model, we use a two-step approach: i) we check if the model reproduces correctly the measured statistics of 


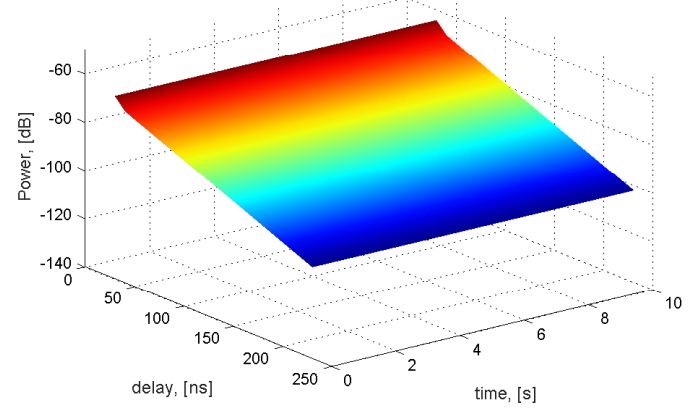

(a) Deterministic PDP, node Rx1

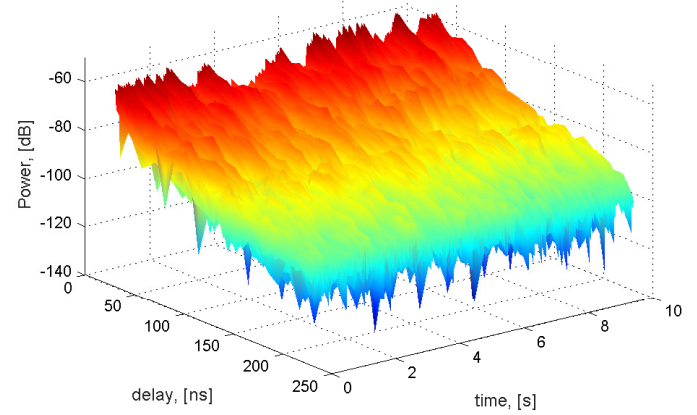

(c) Measured time-variant PDP, node Rx1

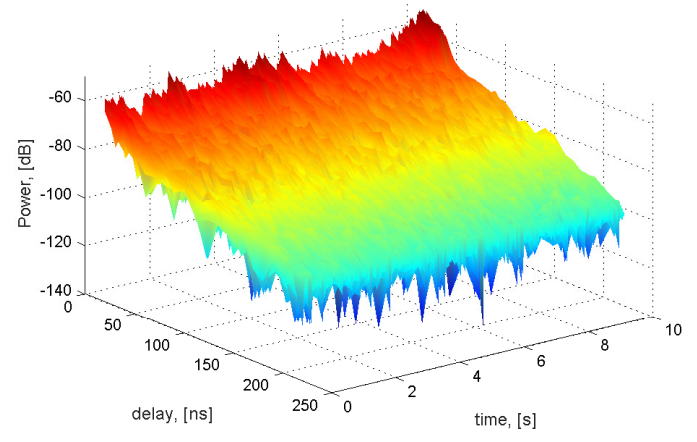

(e) Simulated time-variant PDP, node Rx1

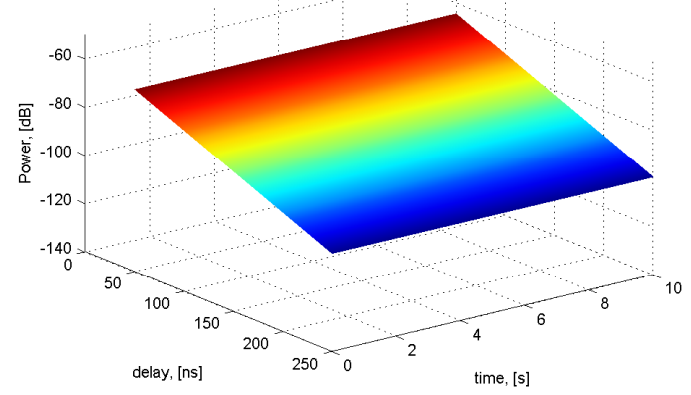

(b) Deterministic PDP, node Rx7

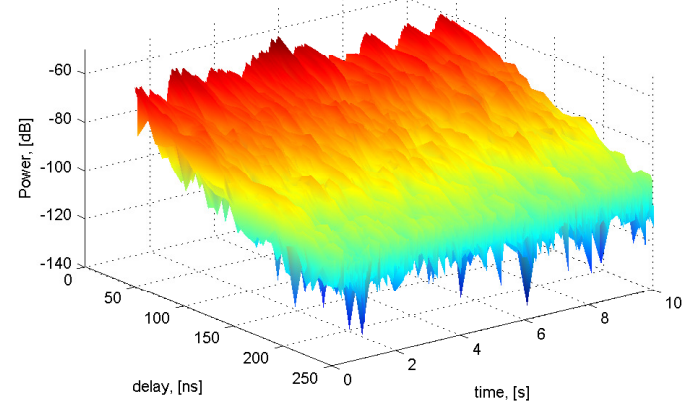

(d) Measured time-variant PDP, node Rx7

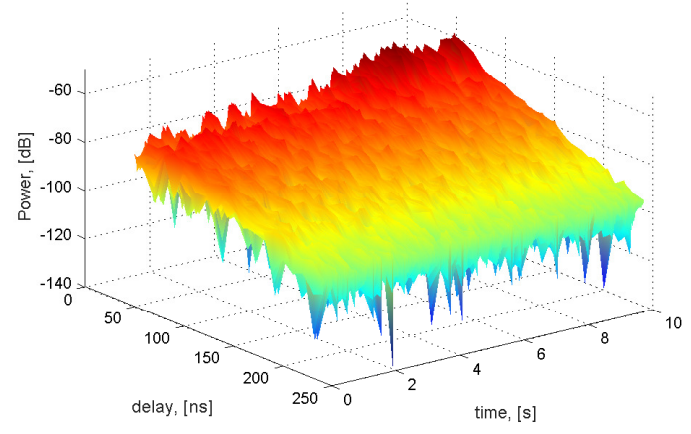

(f) Simulated time-variant PDP, node Rx7

Fig. 7. Deterministic, small-scale fading level, and the total reconstructed PDP. 
the time-delay variant components, ii) we show that parameters derived from the model (but not directly used to parameterize it) agree well with the equivalent measured parameters of the channel.

1) Statistics of Time-Delay Variant Components: Fig. 8 compares the statistics of measured channels and generated channels using the model described in Section IV. To verify the model for different distances, we simulated links between nodes separated by the same distances as we had for measured channels. Next, we compared the cumulative distribution function (CDF) of the generated combination of large- and small-scale fading with the CDF of the same components of the measured set of channels. It is obvious that statistics of simulated channels are in good agreement with the simulated ones.

2) Instantaneous Delay Spread: We define here the instantaneous root-mean-square (rms) delay spread as

$$
\tau_{r m s}(t)=\sqrt{\frac{\int_{0}^{\infty}(\tau-\bar{\tau}(t))^{2} \cdot|h(t, \tau)|^{2} d \tau}{\int_{0}^{\infty}|h(t, \tau)|^{2} d \tau}},
$$

where $\bar{\tau}(t)$ is the mean delay of the instantaneous channel $h(t, \tau)$. The objective of defining the instantaneous rms delay spread is to produce cumulative distribution functions (CDF) of the rms delay values obtained in the dynamic scenario (time domain) for both the measured and predicted PDPs. The comparison of the mean rms values from the measurements and model is listed in Table VI. Parameter $\Delta \tau_{r m s}$ is the difference between the mean predicted and measured rms delay spread values. Relative errors $\Delta \tau_{r m s}=100 \cdot\left(\tau_{r m s}^{\text {exp }}-\tau_{r m s}^{\text {meas }}\right) / \min \left(\tau_{r m s}^{\text {exp }}, \tau_{r m s}^{\text {meas }}\right)$ are also listed in the table. Averaged value of $\Delta \tau_{r m s}$ for all the nodes equals $0.24 \mathrm{~ns}$ (or $1.04 \%$ ). This asymptotic value of the error $\Delta \tau_{r m s}^{a v g}$ is achieved after 20 simulation trials.

The CDFs of rms delay spread values of two nodes are shown in Figs.9 $a$ and $b$. Two cases are demonstrated: Figs.9 a shows CDFs with the largest error $\Delta \tau_{r m s}$, whereas Figs.9 $b$ shows CDFs with $\Delta \tau_{r m s}=0.24 \mathrm{~ns}$, which equals the asymptotic value of the error $\Delta \tau_{r m s}^{\text {avg }}$.

On the one hand, we observe that the rms delay spread value approaches the theoretical reverberation time for the room-to-room scenario, i.e., $\tau=24.80 \mathrm{~ns}$, for increasing $\mathrm{Tx}-\mathrm{Rx}$ distances (e.g. Rx7,8). This shows that the influence of the primary components decreases when the Tx-Rx distance increases and the diffuse fields become prominent. Therefore, the shape of the PDP approaches an exponential decay $\left(\tau_{0} \longrightarrow \tau_{l}\right)$. For node Rx4, we observe the largest error (see Table VI). The possible explanation is that this channel is highly affected by two large metallic cupboards (Fig. 1). 

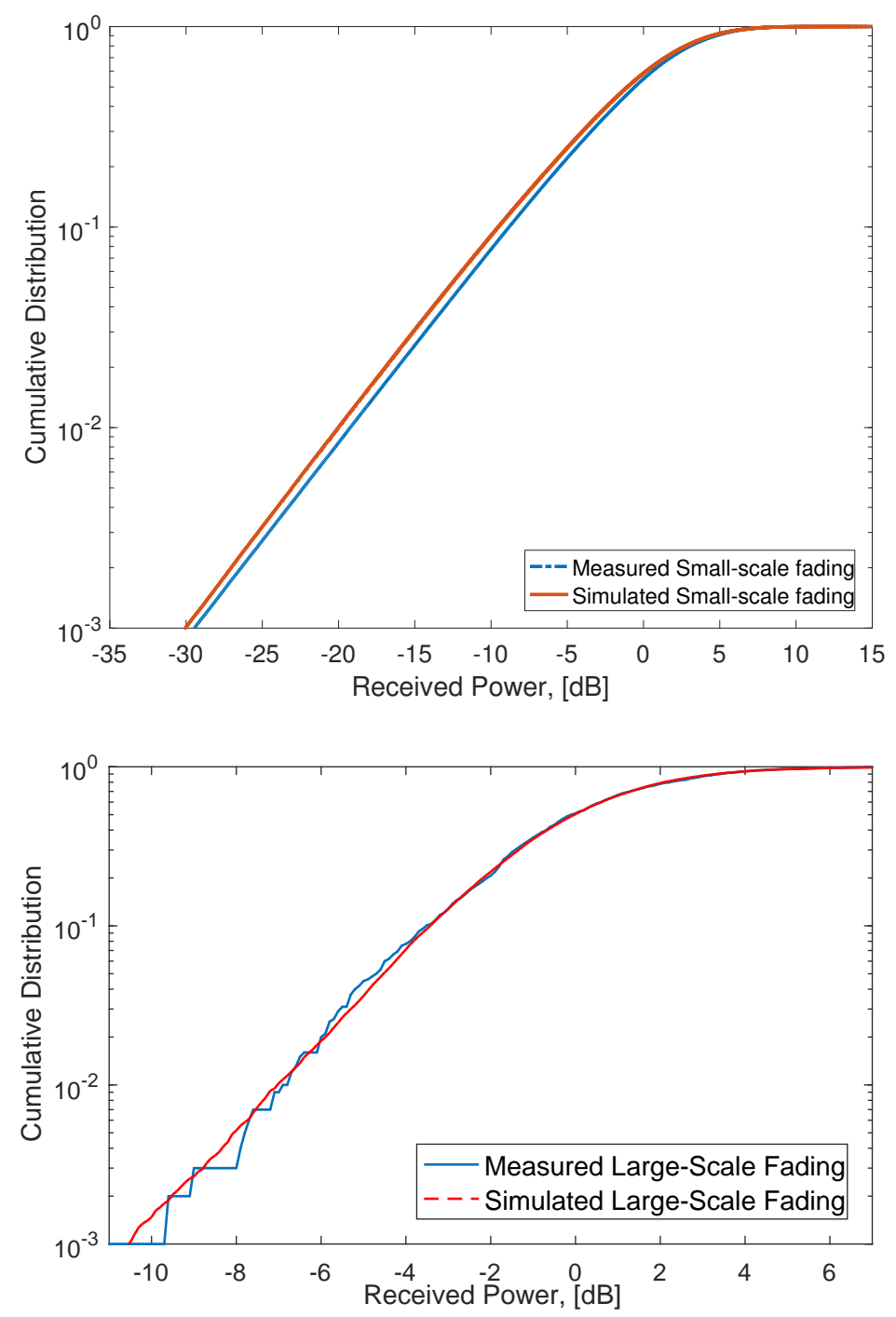

Fig. 8. CDFs of measured and simulated stochastic components (Small-scale fading (top) and Large-scale fading (bottom)

On the other hand, when we compare the cumulative distribution function of the experimental and modeled rms delay spread, we can conclude that the proposed methodology estimates well the PDP in room-to-room scenarios. Measured and simulated CDFs do not agree completely but a reasonably good match in terms of sample mean and sample variance is achieved for all the nodes (except node 4, see Fig. $9 a$ ) with the asymptotic error $\Delta \tau_{r m s}^{a v g}=0.24$ ns. As mentioned above, this large error for node 4 is due to the proximity of this node to large metallic cupboards (see Fig. 1). 
TABLE VI

EXPERIMENTAL AND PREDICTED AVERAGE VALUES OF THE RMS DELAY SPREAD FOR THE DIFFERENT NODES

\begin{tabular}{c|ccccc}
\hline Node & $\mathrm{Rx} 1$ & $\mathrm{Rx} 2$ & $\mathrm{Rx} 3$ & $\mathrm{Rx} 4$ & $\mathrm{Rx} 5$ \\
\hline Distance $(\mathrm{m})$ & 4.9 & 6.5 & 4.6 & 5.6 & 7.4 \\
\hline$\tau_{r m s}^{\text {exp. }(\mathrm{ns})}$ & 20.67 & 23.11 & 19.82 & 23.12 & 22.63 \\
\hline$\tau_{r m s}^{\text {mod. }}(\mathrm{ns})$ & 20.43 & 22.7 & 20.01 & 22.57 & 22.61 \\
\hline$\Delta \tau_{r m s}(\mathrm{~ns})$ & 0.24 & 0.41 & 0.19 & 0.55 & 0.02 \\
\hline$\Delta \tau_{r m s}(\%)$ & 1.17 & 1.81 & 0.96 & 2.4 & 0.09 \\
\hline \hline Node & $\mathrm{Rx} 6$ & $\mathrm{Rx} 7$ & $\mathrm{Rx} 8$ & $\mathrm{Rx} 9$ & $\mathrm{Rx} 10$ \\
\hline Distance $(\mathrm{m})$ & 9.7 & 12 & 12.1 & 9.2 & 7.6 \\
\hline$\tau_{r m s}^{\text {exp. }}(\mathrm{ns})$ & 24.12 & 24.14 & 24.07 & 23.51 & 21.63 \\
\hline \begin{tabular}{c}
$\tau_{r m s}^{m o d .}(\mathrm{ns})$ \\
\hline$\Delta \tau_{r m s}(\mathrm{~ns})$
\end{tabular} & 24 & 24.04 & 24.01 & 23.87 & 22 \\
\hline$\Delta \tau_{r m s}(\%)$ & 0.5 & 0.46 & 0.25 & 1.56 & 1.71 \\
\hline
\end{tabular}

\section{CONCLUSIONS}

We have designed a methodology for the prediction of power delay profiles in room-to-room dynamic scenarios. The success of the method is guaranteed by the predominance of the diffuse scattered fields in the considered environment. Basically, the deterministic part of the method is based on the reverberation time of the environment, which fully describes the diffuse fields and predict the average power level. Moreover, we have proposed models for time-delay largeand small-scale fading. A Hidden Markov model has been used to describe the evolution of the small scale fading statistics and then time-delay fading realizations have been generated using the Young's model [20].

This model enables to describe of the evolution (or change) of the power delay profile in the time domain. The method has been validated against the measurements and good agreement is obtained. Note that the model can be easily extended to double mobility cases by using the five-state HMM described in [16]. Moreover, it has been showed that the distribution of Rician 


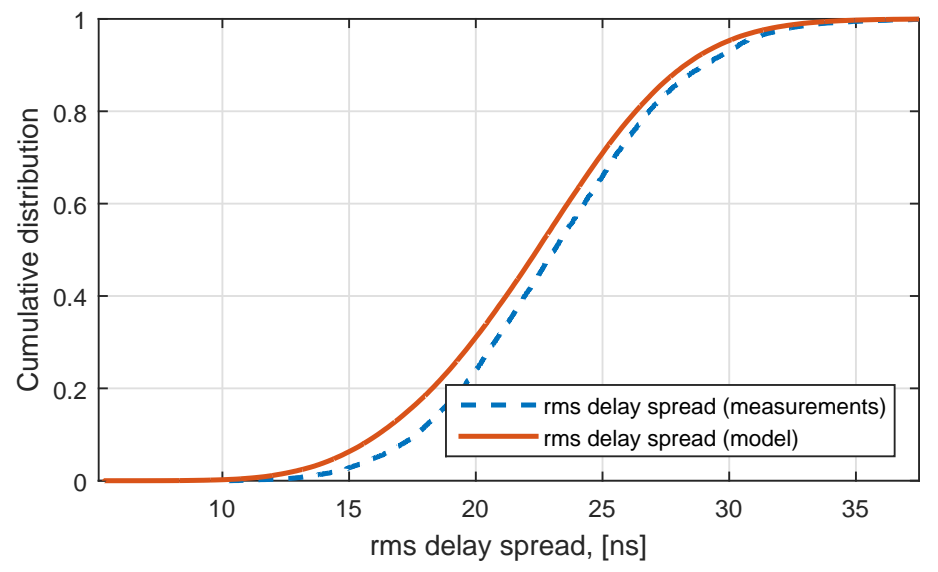

(a) $\mathrm{CDFs}$ of the rms delay spread, node $\mathrm{Rx} 4$ (distance $=5.6 \mathrm{~m}$ )

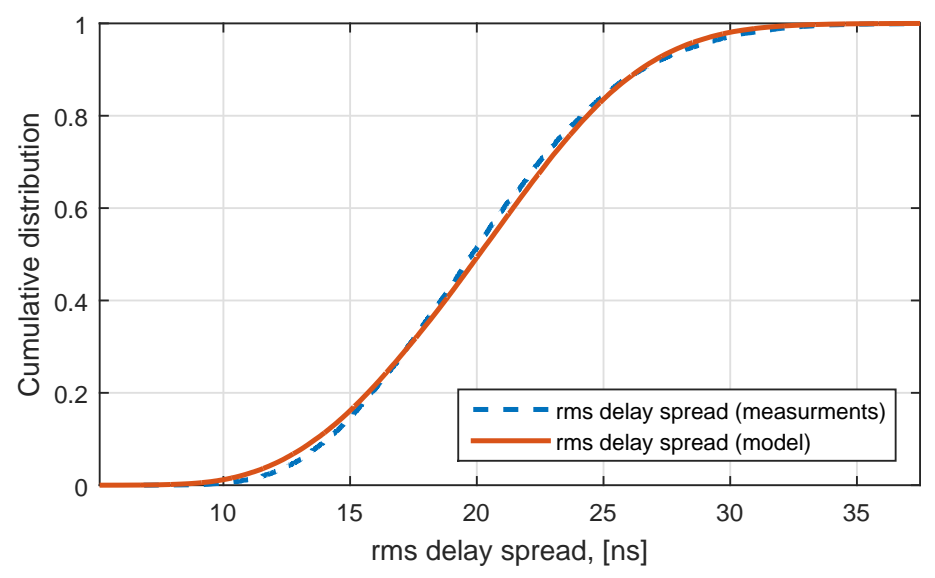

(b) CDFs of the rms delay spread, node Rx1 (distance $=4.9 \mathrm{~m}$ )

Fig. 9. Cumulative distribution function of $\tau_{r m s}$ : measurement versus model

K-factor can be modeled by Extreme Value Distribution as it was done previously in [16].

\section{REFERENCES}

[1] M. Lott, "On the performance of an advanced 3D ray tracing method," in In Proc. of European Wireless and ITG Mobile Communication, 1999.

[2] M. F. Iskander and Z. Yun, "Propagation prediction models for wireless communication systems," IEEE Transactions on Microwave Theory and Techniques, vol. 50, no. 3, pp. 662-673, Mar 2002.

[3] Z. Ji, B.-H. Li, H.-X. Wang, H.-Y. Chen, and T. K. Sarkar, "Efficient ray-tracing methods for propagation prediction for indoor wireless communications," IEEE Antennas and Propagation Magazine, vol. 43, no. 2, pp. 41-49, April 2001.

[4] F. Mani, F. Quitin, and C. Oestges, "Directional spreads of dense multipath components in indoor environments: Experimental validation of a ray-tracing approach," IEEE Transactions on Antennas and Propagation, vol. 60, no. 7, pp. 3389-3396, July 2012. 
[5] T. Rautiainen, R. Hoppe, and G. Wolfle, "Measurements and 3D Ray Tracing Propagation Predictions of Channel Characteristics in Indoor Environments," in 2007 IEEE 18th International Symposium on Personal, Indoor and Mobile Radio Communications, Sept 2007, pp. 1-5.

[6] C. A. 2100, ”Pervasive Mobile and Ambient Wireless Communications”, R. Verdone and A. Zanella, Eds. Springer, 2012.

[7] J. Medbo and J.-E. Berg, "Simple and accurate path loss modeling at $5 \mathrm{GHz}$ in indoor environments with corridors," in Vehicular Technology Conference, 2000. IEEE-VTS Fall VTC 2000. 52nd, vol. 1, 2000, pp. 30-36 vol.1.

[8] J. Kivinen, X. Zhao, and P. Vainikainen, "Empirical characterization of wideband indoor radio channel at 5.3 GHz," IEEE Transactions on Antennas and Propagation, vol. 49, no. 8, pp. 1192-1203, Aug 2001.

[9] K.-G. Tan and S. Denno, "Empirical characterisation of indoor broadband propagation channel," in Personal, Indoor and Mobile Radio Communications, 2003. PIMRC 2003. 14th IEEE Proceedings on, vol. 1, Sept 2003, pp. 950-954 Vol.1.

[10] D. Xu, J. Zhang, X. Gao, P. Zhang, and Y. Wu, "Indoor office propagation measurements and path loss models at 5.25 GHz," in Vehicular Technology Conference, 2007. VTC-2007 Fall. 2007 IEEE 66th, Sept 2007, pp. 844-848.

[11] C. Oestges, D. Vanhoenacker-Janvier, and B. Clerckx, "Channel characterization of indoor wireless personal area networks," IEEE Transactions on Antennas and Propagation, vol. 54, no. 11, pp. 3143-3150, Nov 2006.

[12] C. Oestges, P. Castiglione, and N. Czink, "Empirical modeling of nomadic peer-to-peer networks in office environment," in IEEE 73rd Vehicular Technology Conference (VTC Spring), May 2011, pp. 1-5.

[13] I. Kovacs, P. C. F. Eggers, K. Olesen, and L. Petersen, "Investigations of outdoor-to-indoor mobile-to-mobile radio communication channels," IEEE 56th Vehicular Technology Conference (VTC Fall), vol. 1, pp. 430-434 vol.1, 2002.

[14] J. Nielsen, V. Afanassiev, and J. Andersen, "A dynamic model of the indoor channel," Wireless Personal Communications, vol. 19, no. 2, pp. 91-120, 2001.

[15] C. Oestges, N. Czink, B. Bandemer, P. Castiglione, F. Kaltenberger, and A. Paulraj, "Experimental characterization and modeling of outdoor-to-indoor and indoor-to-indoor distributed channels," IEEE Transactions on Vehicular Technology, vol. 59, no. 5, pp. 2253-2265, Jun 2010.

[16] E. Vinogradov, W. Joseph, and C. Oestges, "Measurement-based modeling of time-variant fading statistics in indoor peerto-peer scenarios," IEEE Transactions on Antennas and Propagation, vol. 63, no. 5, pp. 2252-2263, May 2015.

[17] M. Herdin, “Non-stationary indoor MIMO channels,” Ph.D. dissertation, Technischen Universität Wien, 2004.

[18] L. Bernad, T. Zemen, F. Tufvesson, A. F. Molisch, and C. F. Mecklenbruker, "Time- and Frequency-Varying K -Factor of Non-Stationary Vehicular Channels for Safety-Relevant Scenarios," IEEE Transactions on Intelligent Transportation Systems, vol. 16, no. 2, pp. 1007-1017, April 2015.

[19] Y. Ephraim and N. Merhav, "Hidden Markov processes," IEEE Transactions on Information Theory, vol. 48, no. 6, pp. 1518-1569, Jun 2002.

[20] D. Young and N. Beaulieu, "The generation of correlated Rayleigh random variates by inverse discrete fourier transform," IEEE Transactions on Communications, vol. 48, no. 7, pp. 1114-1127, Jul 2000.

[21] A. Richter, and R. S. Thoma, "Joint maximum likelihood estimation of specular paths and distributed diffuse scattering," in 61st IEEE Vehicular Technology Conference, VTC 2005-Spring, pages 11-15 vol. 1., 2005.

[22] J. B. Andersen, J. O. Nielsen, G. F. Pedersen, G. Bauch, and J. M. Herdin, "Room electromagnetics," IEEE Antennas and Propagation Magazine, vol. 49, no. 2, pp. 27-33, April 2007.

[23] J. B. Andersen, K. L. Chee, M. Jacob, G. F. Pedersen, and T. Kurner, "Reverberation and absorption in an aircraft cabin with the impact of passengers," IEEE Transactions on Antennas and Propagation, vol. 60, no. 5, pp. 2472-2480, May 2012.

[24] H. Kuttruff, Room Acoustics. Spon Press, 2000, London. 
[25] G. Steinböck, T. Pedersen, B. H. Fleury, W. Wang, and R. Raulefs, "Distance dependent model for the delay power spectrum of in-room radio channels," IEEE Transactions on Antennas and Propagation, vol. 61, no. 8, pp. 4327-4340, Aug 2013.

[26] J. Poutanen, J. Salmi, K. Haneda, V. M. Kolmonen, and P. Vainikainen, "Angular and shadowing characteristics of dense multipath components in indoor radio channels," IEEE Transactions on Antennas and Propagation, vol. 59, no. 1, pp. 245-253, Jan 2011.

[27] A. Bamba, W. Joseph, J. B. Andersen, E. Tanghe, G. Vermeeren, D. Plets, J. . Nielsen, and L. Martens, "Experimental assessment of specific absorption rate using room electromagnetics," IEEE Transactions on Electromagnetic Compatibility, vol. 54, no. 4, pp. 747-757, Aug 2012.

[28] O. Franek, J. B. Andersen, and G. F. Pedersen, "Diffuse scattering model of indoor wideband propagation," IEEE Transactions on Antennas and Propagation, vol. 59, no. 8, pp. 3006-3012, Aug 2011.

[29] C. L. Holloway, M. G. Cotton, and P. McKenna, "A model for predicting the power delay profile characteristics inside a room," IEEE Transactions on Vehicular Technology, vol. 48, no. 4, pp. 1110-1120, Jul 1999.

[30] A. Bamba, M. T. Martinez-Ingles, D. P. Gaillot, E. Tanghe, B. Hanssens, J. M. Molina-Garcia-Pardo, M. Lienard, L. Martens, and W. Joseph, "Experimental Investigation of Electromagnetic Reverberation Characteristics as a Function of UWB Frequencies," IEEE Antennas and Wireless Propagation Letters, vol. 14, pp. 859-862, 2015.

[31] A. Zajic, G. Stuber, T. Pratt, and S. Nguyen, "Wideband MIMO Mobile-to-Mobile Channels: Geometry-Based Statistical Modeling With Experimental Verification," IEEE Transactions on Vehicular Technology, vol. 58, no. 2, pp. 517-534, Feb 2009.

[32] B. Fleury, M. Tschudin, R. Heddergott, D. Dahlhaus, and K. Ingeman Pedersen, "Channel parameter estimation in mobile radio environments using the SAGE algorithm," IEEE Journal on Selected Areas in Communications, vol. 17, no. 3, pp. 434-450, Mar 1999.

[33] J. Christmas and R. Everson, "Robust Autoregression: Student-t Innovations Using Variational Bayes," IEEE Transactions on Signal Processing, vol. 59, no. 1, pp. 48-57, January 2011.

[34] G. Matz, "Doubly underspread non-wssus channels: analysis and estimation of channel statistics," in 4th IEEE Workshop on Signal Processing Advances in Wireless Communications - SPAWC 2003, June 2003, pp. 190-194.

[35] A. Ispas, C. Schneider, G. Ascheid, and R. Thom, “Analysis of the Local Quasi-Stationarity of Measured Dual-Polarized MIMO Channels," IEEE Transactions on Vehicular Technology, vol. 64, no. 8, pp. 3481-3493, Aug 2015.

[36] L. Bernado, T. Zemen, A. Paier, J. Karedal, and B. H. Fleury, "Parametrization of the local scattering function estimator for vehicular-to-vehicular channels," in 2009 IEEE 70th Vehicular Technology Conference Fall, Sept 2009, pp. 1-5.

[37] A. Paier, T. Zemen, L. Bernadó, G. Matz, J. Karedal, N. Czink, C. Dumard, F. Tufvesson, A. F. Molisch, and C. F. Mecklenbräuker, "Non-WSSUS vehicular channel characterization in highway and urban scenarios at $5.2 \mathrm{GHz}$ using the local scattering function," International ITG Workshop on Smart Antennas (WSA), pp. 9 - 15, February 2008.

[38] C. Patel, G. Stuber, and T. Pratt, "Simulation of Rayleigh-faded mobile-to-mobile communication channels," IEEE Transactions on Communications, vol. 53, no. 11, pp. 1876-1884, Nov 2005. 


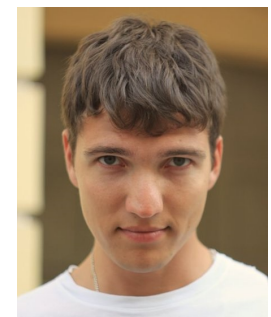

Evgenii Vinogradov was born in Yugorsk, Russian Federation (1986). He received the Dipl. Engineer degree in Radio Engineering and Telecommunications from Saint-Petersburg Electrotechnical University "LETI" (ETU), Saint-Petersburg (Russian Fed.), in 2009. After several years of working in the field of mobile communications, he joined Institute of Information and Communication Technologies, Electronics and Applied Mathematics (ICTEAM) in the Universite catholique de Louvain (UCL) in 2013, where he obtained his $\mathrm{PhD}$ degree in 2017. His doctoral research projects interests focused on radio propagation and wireless channel modeling. In 2017, Evgenii joined the electrical engineering department at KU Leuven (Belgium) where he is working on wireless communications with Unmanned Aerial Vehicles.

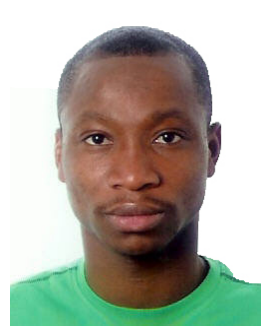

Aliou Bamba was born in Daloa, Ivory Coast, on December 4, 1984. After graduating as an engineer in Mobile Radio Engineering of the National Institute of Posts and Telecommunications, Rabat, Morocco, in 2009, he received the Masters degree in advanced techniques for mobile communications from the University of Lille, France, in 2010. From August 2010 to June 2015, he was a Research Engineer in the WiCa group of Prof. L. Martens, Department on Information Technology (INTEC), Ghent University, Ghent, Belgium. The researches led to the Ph. D degree in June 2015. In 2016-2017, he worked as a research engineer at CEA-LETI Grenoble, France, on mmMAGIC (mm-Wave based Mobile Radio Access Network for 5G Integrated Communications) project. Since 2017, Aliou is assistant at Ecole Supérieure Africaine des TIC (ESATIC), Abidjan, Cote d'Ivoire and his research interests include wireless channel measurements and modeling, human RF absorption due to electromagnetic fields, and millimeter-wave progagation.

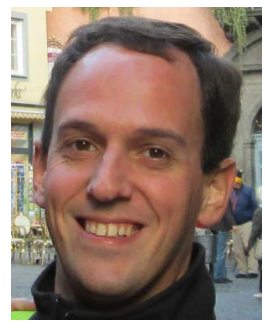

Wout Joseph (M'05 - SM'12) was born in Ostend, Belgium, on October 21, 1977. He received the M. Sc. degree in electrical engineering and the Ph.D. degree from Ghent University (UGent), Ghent, Belgium, in 2000 and 2005, respectively. From September 2000 to March 2005, he was a Research Assistant with the iMinds/UGent-INTEC (Ghent University - Department of Information Technology). During this period, his scientific work was focused on electromagnetic exposure assessment. His research work dealt with measuring and modeling of electromagnetic fields around base stations for mobile communications related to the health effects of the exposure to electromagnetic radiation. This work led to the Ph.D. degree. Since April 2005, he has been a Postdoctoral Researcher with iMinds-UGent/INTEC. From October 2007 to October 2013, he was a Postdoctoral Fellow of Research Foundation- Flanders (FWO-V). Since October 2009, he has been a Professor in the domain of experimental characterization of wireless communication systems. His professional interests are electromagnetic field exposure assessment, propagation for wireless communication systems, antennas, and calibration. Furthermore, he specializes in wireless performance analysis and quality of experience. 


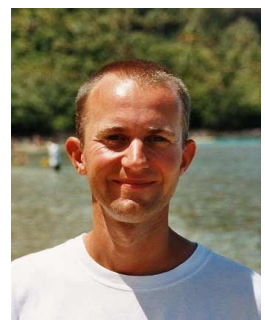

Claude Oestges received the M.Sc. and Ph.D. degrees in Electrical Engineering from the Université catholique de Louvain (UCL), Louvain-la-Neuve, Belgium, respectively in 1996 and 2000. In January 2001, he joined as a post-doctoral scholar the Smart Antennas Research Group (Information Systems Laboratory), Stanford University, CA, USA. From January 2002 to September 2005, he was associated with the Microwave Laboratory UCL as a post-doctoral fellow of the Belgian Fonds de la Recherche Scientifique (FRS-FNRS). Claude Oestges is presently Professor with the Electrical Engineering Department, Institute for Information and Communication Technologies, Electronics and Applied Mathematics, UCL. He also currently serves as an Associate Editor for the IEEE Transactions on Antennas and Propagation and the IEEE Transactions on Vehicular Technology. $\mathrm{He}$ is the author or co-author of three books and more than 180 journal papers and conference communications, and was the recipient of the 1999-2000 IET Marconi Premium Award and of the IEEE Vehicular Technology Society Neal Shepherd Award in 2004 and 2012. 